\title{
New Approach to
}

\section{Heart Sound Screening in Children}

\section{by Analog-Digital Circuitry}

\author{
ROBERT E. DURNIN, M.D., HARVEY F. GLASSNER, B.A. \\ CLINTON O. JORGENSEN, B.S.E.E., and DONALD C. FYLER, M.D.
}

$\mathrm{T}$ HIS REPORT is concerned with a new approach to the timing and interpretations of heart sounds by analog-digital circuitry. It presents the background development of this concept as well as an initial evaluation of the feasibility unit.

Many observers feel a significant number of children have undiagnosed cardiac lesions. Early detection of congenital heart disease is important since earlier surgical intervention is being advocated for many operable congenital lesions. Identification of unrecognized rheumatic heart disease has marked significance in prevention of recurrences, prophylaxis, and clinical followup. The uncovering of these unrecognized lesions has led to the emphasis on yearly school examinations. A well-supervised heart-sound screening program could be a useful adjunct to an established school and community health program. Such a program would be complementary to a health program centered around routine examinations by physicians.

Significant murmurs, and to a less extent, abnormal heart sounds, are the hallmark of heart disease in children. Many elaborate and valuable field studies have been reported regarding methodology of screening and evaluation of taped heart sounds (1-3), and present techniques and equipment continue to have extensive field trials. The development of an analogdigital analyzing unit to scan heart sound signatures appeared as a reasonable approach in an attempt to differentiate normal heart sounds and murmurs from abnormal sounds and murmurs.

Clinical results of present and newer techniques for screening will determine the applicability and advisability of such instrumentational techniques as part of a strong community-oriented health program.

\section{Method}

The development of the analog-digital computer was divided into four work phases: 1 . data acquisition, 2. data analysis, 3. circuitry design and elaboration, and 4. clinical evaluation of the feasibility unit. The study concerned only children of the ages 3 to 17 years.

\section{Phase 1-Data Acquisition}

Phase 1 was concerned with the collection of sufficient heart-sound data to be representative of the range of normal and abnormal (congenital and rheumatic) sounds observed clinically.

Dr. Durnin is a fellow in cardiology and assistant in pediatrics, cardiology division, department of pediatrics, Los Angeles Childrens Hospital and the University of Southern California School of Medicine, Los Angeles. Mr. Glassner is a staff scientist and Mr. Jorgensen an electrical engineer with the Thiokol Chemical Corp. of Los Angeles. Dr. Fyler is chief of cardiology, Los Angeles Childrens Hospital, and associate professor of pediatrics, University of Southern California School of Medicine, Los Angeles. 
Heart sounds of a total of 130 subjects, ages 3 through 17 years, were recorded. A trailer was equipped with a Minneapolis Honeywell 8100 seven-channel FM tape recorder. Heart sounds were recorded at four precordial areas simultaneously-apex, fourth left intercostal space (4LIS), second left intercostal space (2LIS), and second right intercostal space (2RIS) by means of modified Altec-Lansing No. 21 capacitance microphones. Tektronix low-level preamplifiers type FM 122 fed the tape recorder appropriate signal levels from all sensors. Simultaneously, voice identification, ECG (lead II), and respiratory phase were recorded (fig. 1). All recordings were done with patients in the supine position.

The cardiologist associated with all clinical phases of this study examined all 130 patients. Each patient had a routine ECG (electrocardiogram) and chest X-ray. Phonocardiograms were recorded for all children who had abnormal heart sounds. Sixty-four patients specifically selected from the cardiac clinic of Los Angeles Childrens Hospital were classified by the cardiologist as having representative abnormal auscultatory findings. Forty-five patients of the abnormal group had congenital heart disease, of whom 39 had their diagnosis defined at cardiac catheterization or later verified at surgery. The other six children had clinical diagnoses of congenital heart disease. These 130 children provided a wide range of representative congenital heart defects:

Ventricular septal defect.

Ventricular septal defect and pulmonary stenosis

Ventricular septal defect with pulmonary vascular disease

Pulmonary stenosis

Aortic: stenosis

Atrial septal defect

Patent ductus arteriosus

Pulmonary stenosis and pulmonary insufficiency-

Aortic stenosis and aortic insufficiency

Coarctation of the aorta.

Tetralogy of Fallot

Tetralogy of Fallot with absence of the pulmonary valve

Transposition of the great vessels

Corrected transposition with pulmonary stenosis and ventricular septal defect.

Congenital mitral insufficiency
Eight patients of the abnormal sound group had rheumatic heart disease. Five had mitral insufficiency, two aortic insufficiency, and one patient had mitral insufficiency and stenosis. One child had congenital ventricular bigeminy with an associated loud aortic ejection murmur. Ten children included in the abnormal sound group had grade 2 or greater systolic murmurs on Levine's (4) scale of 6 grades, murmurs that were judged to be suspicious but not associated with heart disease. These 10 children have been routinely followed in the cardiac clinic; they have normal X-rays, ECG's, blood pressures, and hemograms.

Sixty-six children of physicians and employees were recorded for baseline normal heart-sound data. Within this group, 56 children had no murmurs; 10 had grade 1 systolic murmurs. This group of children had normal ECG's and X-rays.

\section{Phase 2-Data Analysis}

The objective of phase 2 was to determine those distinguishing characteristics of the heart sounds collected on tape as a means of discriminating between the normal and abnormal sound data. Those sound signatures representative of the normal and abnormal patterns were to be delineated by analysis circuitry.

The taped sounds of all patients for all four precordial areas were played back via analysis circuitry which considered the parameters of frequency, amplitude, and time, independently and as they are interrelated. Conversion of this analog information into a digital printout aided in establishing precisely those characteristics that would lend themselves to circuitry development (fig. 2).

\section{Phase 3-Circuitry Development}

Electronic circuitry was conceived, designed, and developed by Glassner and Jorgensen to detect thoso sound patterns defined in phase 2. A beat-by-beat (that is, in real time) analysis was selected as a means of eliminating those errors inherent in an integrating approach. Subtle variations that exist in individual cardiac cycles may be missed when an integrating approach is used. The analog-digital circuitry of the feasibility unit was designed to analyze specific frequencies and energies of murmurs 
Figure 1. Phase 1-Data Acquisition

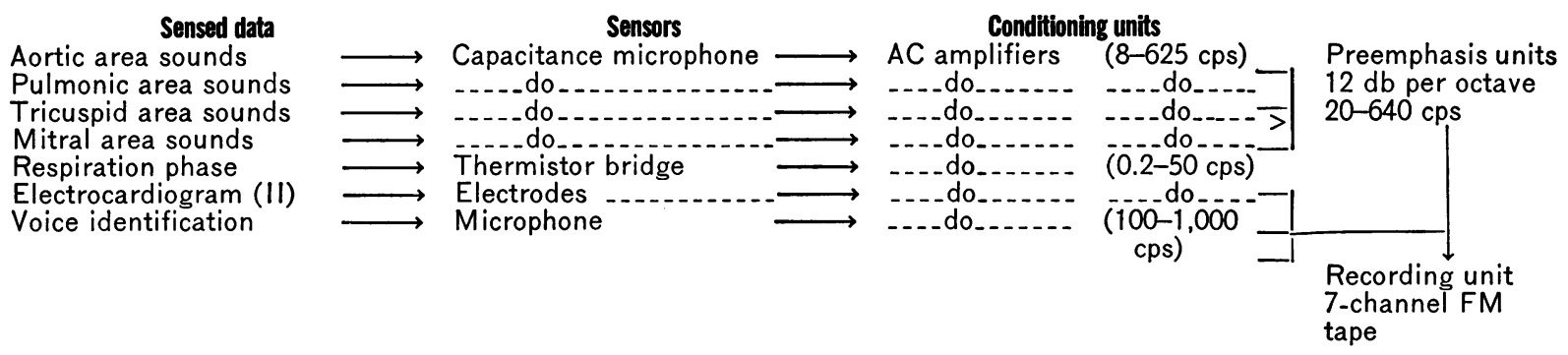

Normal and abnormal heart sound data were recorded with a 906 Honeywell 8100 7-channel FM tape recorder. Four precordial areas, respiration phase, ECG and voice identification were recorded simultaneously.

encountered in clinical auscultation $(5-8)$. By attenuation of some frequency bands and accentuation of others, it was possible to reduce to a great degree the unit's sensitivity to the lowfrequency systolic murmurs representative of most functional murmurs. Obviously, duration and amplitude (that is, energy content) factors are in themselves of significance regardless of frequency content. The initial circuitry of the feasibility unit was designed to ignore the sound signatures of specific grade 1 systolic murmurs. A circuit to analyze all consistent systolic murmurs above this level was mandatory. The diastolic circuitry components to be programed were the high- and low-frequency diastolic murmurs, with the normal third heart sound being ignored. A circuit to identify splitting of the second sound (split $\mathbf{S}_{2}$ ) beyond 50 milliseconds was also designed.

The initial unit required two inputs from the patient: a miniature chest microphone, which was moved to each of the four precordial auscultatory areas, and a single ECG leadtwo Sanborn suction cup electrodes placed at the level of the seventh intercostal space in the midclavicular line (fig. 3). The areas selected for evaluation were apex, fourth left, second left, and second right intercostal spaces with the patient in the supine position. The ECG locates each sound within the cardiac cycle. The bipolar timing lead is similar to lead I of a conventional ECG system. The unit's ECG circuitry recognizes one of three wave forms from this bipolar lead : positive deflection (positive $R$ wave), negative deflection (negative $R$ ), or biphasic deflection (biphasic R). A positive $R$ wave is indicated for timing of the unit.
The ECG signal and heart-sound energies are monitored by means of two meters on the front panel of the unit (fig. 3). The heart-sound meter is stationed at the same setting for each patient. The meter maintains a standard sound-input level to the analyzing circuitry and thereby compensates for varying levels of sound obtained because of different chest sizes.

A preselected number of cardiac cycles, for example, 10, 20, are automatically examined on a beat-by-beat analysis basis. These data are displayed on two digital counters. One registers, with every beat of the heart, the number of cycles examined (NCE), and the other registers only those cycles containing sound patterns which are considered outside the programed normal limits (ONL) of the unit's circuitry. A numerical ratio is thus presented indicating the number of cycles outside the programed normal limits (ONL) to the number of cycles examined (NCE). An ONL: NCE ratio of 60 percent or greater for any one of the analyzing circuits (namely, systole, diastole, split second sound) was considered outside the programed normal limits of the unit. This ratio was determined from the unit's analysis of the data obtained from the initial tapings of normal and abnormal heart sounds and was based on the physiological variability of sounds with respirations and the normal variations observed when analysis is done on a beat-by-beat basis.

Auxiliary displays simultaneously present the basis for considering rejection of any cycle outside normal limits by indicating, with every beat, systolic involvement, diastolic involvement, split second sound (greater than .05 second), or any combination of these. Heart rates 
less than 60 or greater than 130 are indicated by an appropriate display. Figure 3 illustrates one of the first transistor feasibility units (PhonoCardioScan), which weighs 18 pounds and is the size of a standard attaché case.

\section{Phase 4-Clinical Evaluation}

The PhonoCardioScan feasibility unit was evaluated on 708 subjects ages $31 / 2$ years to 17 years; 8 infants were also evaluated. Of these subjects, 438 had normal or functional sounds and 270 had heart disease or suspicious heart sounds. Each child was examined in succession by a technician using the feasibility unit and by a cardiologist. The technician was unaware of the patient's clinical diagnosis. Two cardiologists evaluated 150 of the 270 children in the abnormal-sound group.

This group of 708 subjects was drawn from the cardiac clinic, medical-surgical wards, and a school population. No specific case selection was made. The abnormal cases reflected a cross-section of the congenital and rheumatic lesions seen in the cardiac clinic and in the hospital population. Many of the patients seen in the cardiac clinic were known to the examin- ing cardiologist. The main emphasis was placed on whether the unit's analysis of the heart sounds and murmurs corresponded with the physician's interpretations of the sounds and their clinical significance. Emphasis was placed first on whether the unit's analyzing circuitry judged the heart sounds normal or abnormal. Normality and abnormality as defined by this analyzing device are based solely on the unit's analysis of heart sounds and the wave shape of the QRS complex of the ECG. If a case was rejected by the analyzing unit, then specific areas of rejection (systole, diastole, split $\mathrm{S}_{2}$ ) were compared for accuracy against the cardiologist's interpretations. Characteristics of heart sounds not evaluated by the unit could not be compared.

The aims of the initial clinical evaluation were as follows :

1. To determine whether the analyzing circuitry could, as proposed, scan and interpret heart sounds in real time.

2. To determine whether the analyzing circuitry could separate normal heart sounds and obvious functional sounds from abnormal and suspicious sounds.

3. To correlate and compare physician's aus-

\section{Figure 2. Phase 2-Data Analysis}

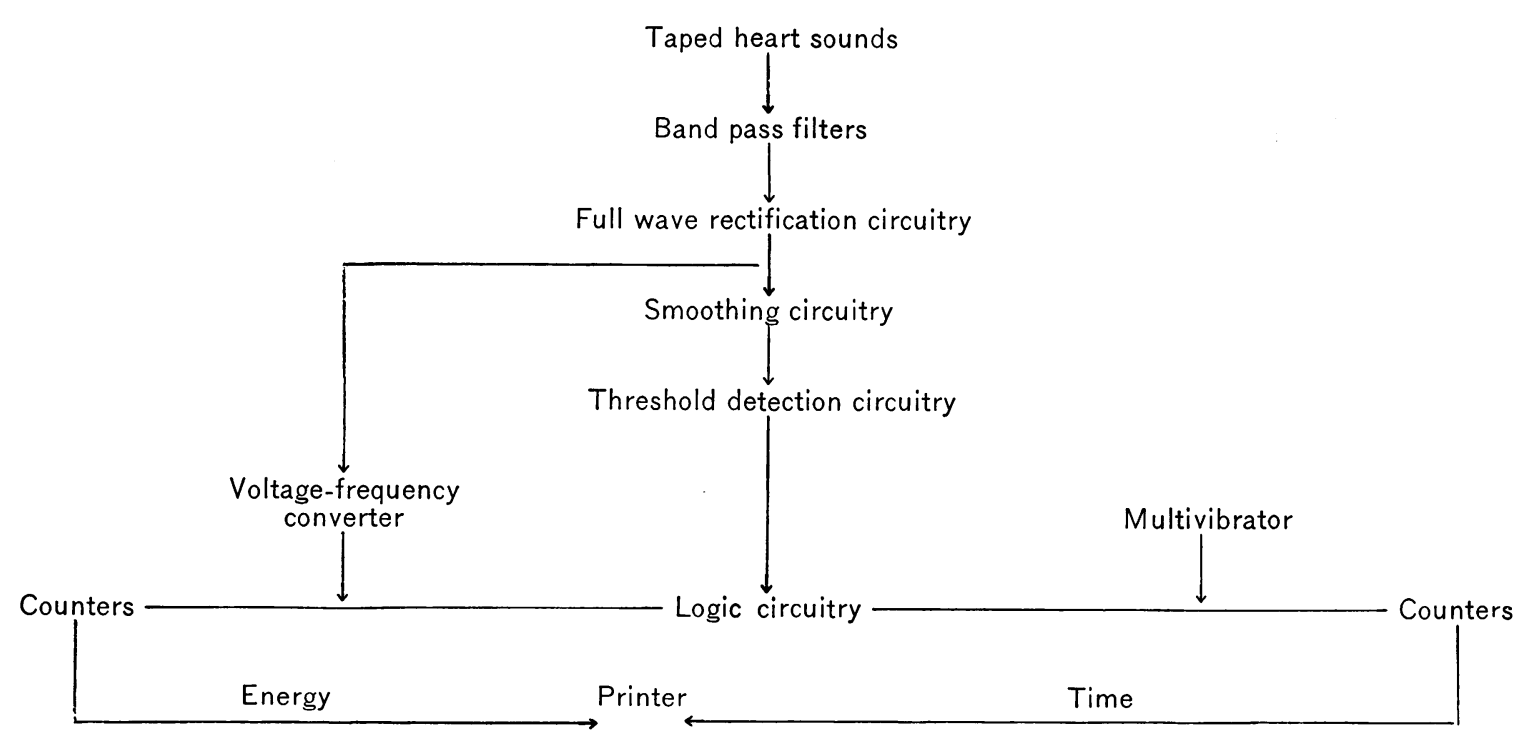

Taped heart sounds were converted from analog information to a digital printout. These data were then analyzed to determine sound characteristics that would lend themselves to circuitry development. 


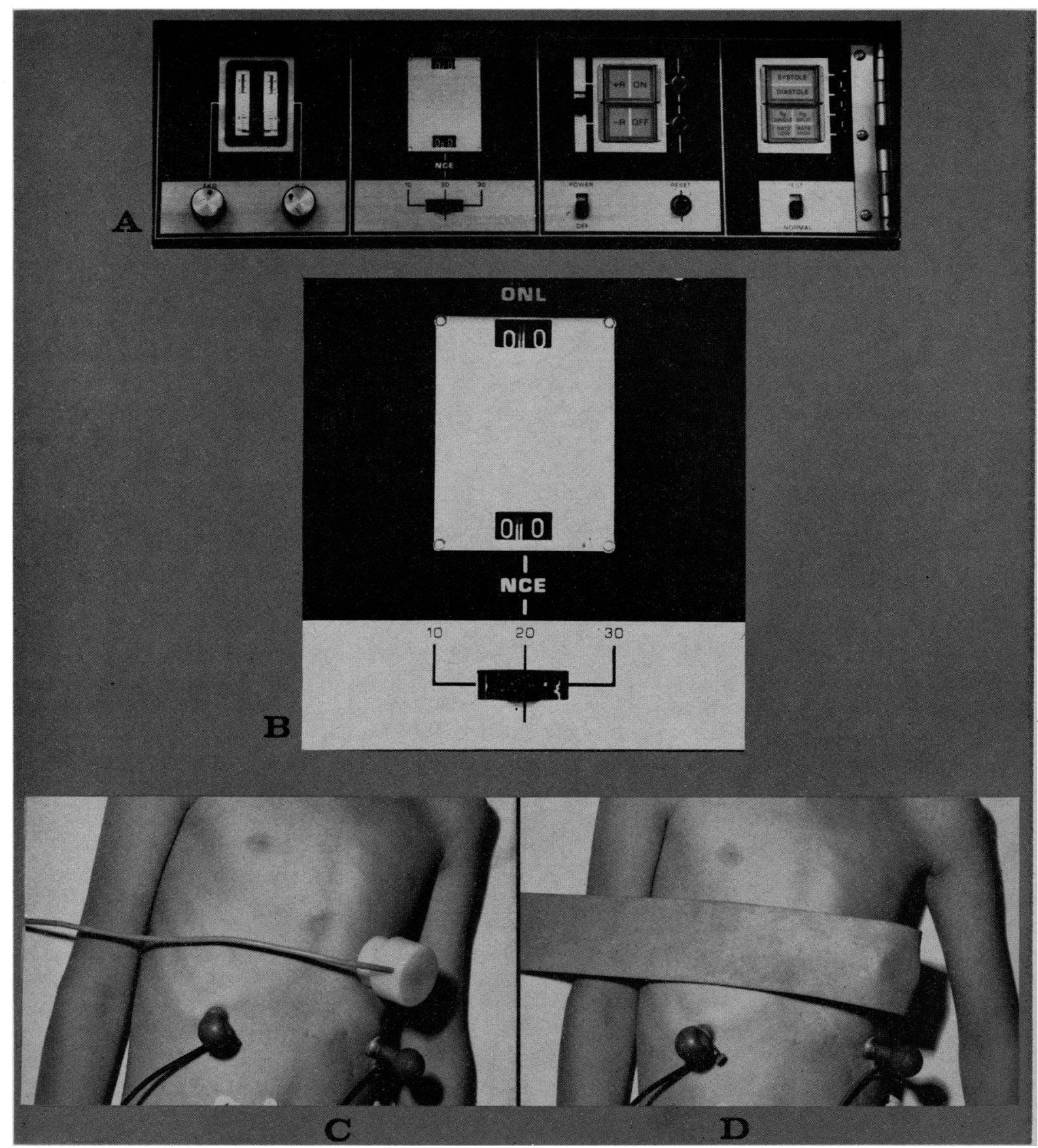

Figure 3. Analog-digital analyzing unit

A.-Front panel of unit. From left to right are the sound and ECG monitoring meters, digital data display counters, ECG display and counter button, and indicators for rejection (systol, diastole, and so forth). The power switch and counter reset button are located at bottom of the third small panel. B.Digital counters. ONL displays the number of cycles outside the programed normal limits, and
NCE indicates the number of cardiac cycles examined. Separate counters (not shown) display the breakdown of ONL (for example, 10) into specifics. The lower button is set to either 10,20 , or 30 for the number of cycles to be examined. C.-Patient with ECG electrodes and microphone in place. D.Microphone held in place by vinyl belt. 
cultatory impression against the systolic, diastolic, and split $S_{2}$ analysis of the unit.

4. To determine the validity of the 60 percent reject ratio.

5. To determine the accuracy of the unit in recognizing abnormal sounds and the percent of false negatives and false positives in the population screened.

6. To determine the unit's applicability as a heart sound screener.

7. To evaluate ancillary displays, heart rate, and ECG polarity.

Since the ECG timing lead is in most instances similar to a conventional lead $\mathrm{I}$, the finding of a negative $R$ or biphasic $R$ would presumptively suggest right axis deviation, right ventricular hypertrophy, or possibly combined ventricular hypertrophy. This assumption, we anticipated, would be answered during the initial clinical evaluation phase. Biphasic $\mathbf{R}$ and negative $\mathbf{R}$ would then also serve as a basis for rejection regardless of the recorded heart sound ratio.

The information obtained from initial evaluation would aid in determining: $(a)$ dependency and stability of the initial unit's analyzing circuitry, $(b)$ deficiencies of one or more specific circuits, $(c)$ advisability of circuitry change or modification, $(d)$ desirability of further circuitry design, and $(e)$ reaction of the patient and his effect, if any, on machine operation. In the early clinical phase, evaluation of the second right intercostal area was difficult because of bronchial respiration noise and movement; deletion of this examining area did not alter the overall accuracy of the unit.

Thus, during the initial clinical evaluation, analog computer findings of an ONL: NCE ratio (the ratio between number of cycles outside programed normal limits and number of cycles examined) of 60 percent or greater for systole, diastole, or $\mathrm{S}_{2}$ analysis or any combination of such findings were interpreted as a basis for rejection and classification of the heart sounds as abnormal. The finding of biphasic $R$ or negative $\mathrm{R}$ by the ECG timing lead would indicate a basis for rejection.

Results for normal group. In the normal group, 437 patients had noncardiac diagnoses and 1 child had old primary endomyocardial disease:
Diagnosis

Total normal group evaluated

Normal heart sounds_-_-_-_-_-_-_-_-_-_-_ 336

Normal heart sounds (endomyocardial disease)

Functional murmurs 101

Evaluation by cardiologist and unit:

Normal or functional sounds-cardiologist_-- 438

Recorded as within normal limits by the computer

412

Recorded as outside programed normal limits by the computer (false-positives) -...-..-Grade 1-2 functional murmurs Mesocardia with functional murmur...-Asthma and functional murmur.-_-_-_-_-Uncooperative

Accentuated third heart sound.---.---Rejected because of unit timing problem.---

Recorded biphasic $R$ wave on ECG timing lead

Wide splitting of second sound.

Three hundred and one of these children came from a school population having yearly physical examinations and reliable medical histories. The remaining 137 children were inpatients from a hospital population with noncardiac medical-surgical diagnoses. Eighty-five inpatients had normal routine chest $\mathrm{X}$-rays.

This group of 438 children were judged to have normal heart sounds or functional (insignificant) murmurs by the cardiologist. The femoral pulses were also checked by the cardiologist. Three hundred thirty-seven children had normal heart sounds; 101 had grade 1-2 systolic murmurs considered to be functional. Four hundred and twelve children were passed by the analyzing circuitry as having sounds that were not outside the programed normal limits, a finding in agreement with the cardiologist's interpretations. Twenty-six children were analyzed as outside the normal limits (ONL) of the unit's analyzing circuitry and constituted false positives. Twenty-two were rejected by the heart-sound analyzing circuit. Four were rejected by the ECG circuitry solely because of a biphasic $R$ wave; these four patients had normal ECG's and heart sounds. Eight children were rejected at the 2RIS area, which has been deleted from routine analysis. Since their heart sounds were normal in other areas, they were not called ONL and are not considered in the false-positive group.

Twenty-six false positives were recorded by the unit. The child with primary endomyo- 
cardial disease and normal heart sounds constituted the one false negative. The cardiologist felt that none of this group had heart-sound abnormalities that suggested the possibility of heart disease. The one child with mesocardia would probably suggest eccentricity of cardiac position, but his heart sounds would be judged normal. Overall, in this normal group the unit called 5.9 percent false positives as compared to the cardiologist.

Results for abnormal group. A total of 270 children with heart disease or suspicious murmurs were evaluated by the unit's analyzing circuitry. These children were not part of the group used for the data-acquisition phase. The diagnostic categories encountered were as follows :

\section{Diagnosis}

Patients

Congenital heart disease 244

Suspicious murmurs 18

Ventricular septal defect.

Ventricular septal defect with pulmonary hypertension.

Ventricular septal defect with mild pulmonary stenosis

Ventricular septal defect with aortic insufficiency

Ventricular septal defect with coarctation.--

Ventricular septal defect with pulmonary hypertension, mitral stenosis

Postoperative ventricular septal defect and atrial septal defect

Postoperative ventricular septal defect.

Closing ventricular septal defect or functional murmur

Patent ductus arteriosus

Pulmonic stenosis

Postoperative pulmonic stenosis

Atrial septal defect (secundum)

Pulmonic stenosis, atrial septal defect.---_---

Atrial septal defect with pulmonary hypertension.

Aortic stenosis

Postoperative aortic stenosis

Aortic stenosis and aortic insufficiency

Congenital aortic insufficiency

Coarctation of the aorta

Postoperative coarctation

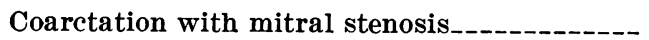

Endocardial cushion defect (ostium primum) -

Pulmonary insufficiency

Tetralogy of Fallot

Tetralogy of Fallot with operative shunt

Postoperative tetralogy (total correction) -----

Aortico-pulmonary window with pulmonary hypertension

Endocardial fibroelastosis with mitral in-

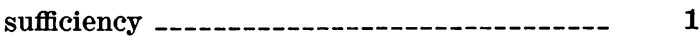

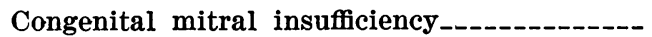

Hurler's syndrome - mitral insufficiency-..-.-

Postmitral valve replacement (congenital mitral insufficiency)

Coronary arterio-venous fistula

Ebstein's anomaly-

Single ventricle with pulmonic stenosis

Transposition of the great vessels

Transposition of the great vessels (post Senning) -

Corrected transposition with ventricular septal defect

Tricuspid atresia

Truncus arteriosus.

Marfan's syndrome

Dextrocardia, ventricular septal defect._-_---

Dextrocardia, tetralogy of Fallot_-_.-_-_--.--

Hemic murmurs

Rheumatic heart disease

Mitral insufficiency

Mitral insufficiency and aortic insufficiency---

Aortic insufficiency.

Postmitral valve prosthesis (Starr valve) -.--

Rhythm and conduction disturbances

Complete heart block

Complete right-bundle-branch block

Total

270

One hundred and two children in this group had had catheterization. All 270 had abnormal heart sounds as judged by the cardiologist's auscultatory interpretations; 261 of the 270 had systolic murmurs; 84 of the patients had systolic murmurs that were grade 2 on scale of 6,56 were grade $2-3$, and 121 had murmurs recorded as grade 3 or greater.

Two hundred sixty-four of this group were analyzed by the unit as having abnormal heart sounds with ONL ratios of 60 percent or greater :

Diagnosis

Patients

By cardiologist: abnormal heart sounds and murmurs -

By computer-number evaluated._-_-_-- 270

Recorded as outside programed normal limits_ 264

Recorded as within programed normal limits (false negatives)

Tetralogy of Fallot.

Aortico-pulmonary window with pulmonary vascular disease.

Ventricular septal defect with pulmonary vascular disease.

Marfan's syndrome with aortic insufficiency

Atrial septal defect with pulmonary hypertension

Ventricular septal defect. 
In specific areas of analysis, the unit was most accurate in screening systolic sounds. One child with a patent ductus arteriosus was rejected by the diastolic circuitry, but the systolic murmur was not consistently recognized. Forty-five patients had negative $R$, and 19 had a biphasic $R$ wave recorded by the ECG lead circuitry.

The areas of disagreement between the cardiologist's interpretations and the unit were the diastolic sounds. Four of seven semilunar insufficiency murmurs were initially missed by the unit although it did reject the four children for an accompanying systolic murmur. During the clinical phase, the diastolic circuitry was reworked to increase its capabilities with regard to high-frequency sounds. These additions to the diastolic circuitry had no effect on the overall analysis of the unit or on analysis of the previously run normal and abnormal cases as determined by parallel runs of the same patients with both circuits. Of 12 insufficiency murmurs analyzed by the unit since the additions, the unit has recognized 9 . The diastolic murmurs were recorded as grade $2-3$ intensity. One was called grade 1-2. Diastolic rumbles and the diastolic components of continuous murmurs were consistently recorded as abnormal by the unit. Those modalities of heart sounds such as accentuated or single second heart sounds were not compared.

Heart sounds of six children in the abnormal group were analyzed as normal by the unit's heart-sound analysis circuitry. These six cases included a severe tetralogy with a grade 1 murmur, an aortico-pulmonary window with pulmonary vascular obstruction, and a ventricular septal defect with pulmonary vascular obstruction. The latter two had no murmurs. One child with an atrial septal defect and pulmonary hypertension had a grade 1-2 ejection murmur. These four children did record a biphasic $\mathrm{R}$ or negative $R$ by the ECG circuitry. One child with Marfan's syndrome had a grade 1 aortic insufficiency murmur and was missed, as was a child with a grade 2 ventricular septal defect murmur (fig. 3). These misses constituted a 2 percent false-negative finding for this group of 270 patients. If the four children who recorded negative $R$ and biphasic $R$ are not considered in the false-negative group, the overall false-negative finding for this abnormal group is 0.7 percent.

The polarity circuit of the ECG lead was then analyzed to determine the validity of assuming that a biphasic $\mathbf{R}$ or negative $\mathrm{R}$ suggested right axis deviation or right ventricular hypertrophy. Of 64 children with abnormal heart sounds who also had negative $R$ (45) or biphasic $R$ (19), 62 had right ventricular hypertrophy or combined ventricular hypertrophy; 2 had normal ECG's. Three children in the abnormal group who had significant right ventricular hypertrophy recorded a positive $\mathrm{R}$ wave. Four children in the abnormal group, however, were rejected solely on the basis of negative $R$ and biphasic $R$. It appears that additional unexpected information was obtained from this simple timing lead. The timing lead at the present time does not recognize premature beats. These may be considered in later design. The heart rate circuit offered no additional information.

\section{Discussion}

The beat-by-beat method by which this unit analyzes the heart sounds and intervals was specifically chosen as a means of approaching, as closely as possible, the technique used in clinical auscultation. By incorporating into this design subtle variations existing from beat to beat in frequency, the amplitude and time parameters of the sound pattern will not be ignored. The ONL: NCE ratio presented by the unit will reflect these changes. The normal sound pattern may well incorporate a grade 1 murmur varying significantly with respiration and position, and the ONL: NCE ratio indicates this variation. For example, if 20 cycles are examined, a 6:20 ratio may be presented. This ratio indicates that the sound parameters examined exceeded the set programed normal limits 30 percent of the time.

In examining 20 cycles of an abnormal sound pattern, the ONL: NCE ratio may be $16: 20$, suggesting that 4 cycles did not exceed the set normal limits with respect to the frequency, amplitude, and time relationships. This finding can be more clearly observed when a split second sound is analyzed. In many cases, the split varies above and below the circuit's 50 
millisecond reject level, and therefore not all cycles are considered ONL.

The preclinical phase indicated that an ONL: NCE ratio of 60 percent or more should be a basis for rejecting a child. This ratio includes murmurs of greater than grade 1 intensity. Grade 1 short-duration systolic murmurs and some inconsistent grade 1-2 murmurs which are usually unassociated with heart disease gave a ratio of less than 60 percent. There was good correlation between the unit's analysis of systolic sounds as compared to the physician's interpretations.

The areas of disagreement between the cardiologist and unit in diastole were in terms of the low-intensity insufficiency murmurs and in interpretations of the accentuated and narrowly split second sound. The latter two could not be analyzed by the unit. Overall, 67 percent of the insufficiency murmurs presented to the analyzing circuitry were recognized. Seventyfive percent were recognized after the capabilities of the diastolic circuitry were increased. Concern over the low-intensity insufficiency mumurs and the unit's deficiency in this area of analysis has led to continuing improvement of the diastolic circuitry. Presently, analysis of diastole at 3LIS with held expiration is being tested in an attempt to better define the lowenergy diastolic insufficiency murmurs. Although semilunar valve insufficiency murmurs are clinically in the minority, their significance need not bo emphasized. This area (3LIS) may well be incorporated as a screening area along with apex, 4LIS, and 2LIS. Wide splitting of the second sound (split $\mathrm{S}_{2}$ ) correlated well with the clinical and phonocardiographic findings in the 15 cases in which this finding was noted.

Additional information was obtained from the ECG leads. Circuitry within the machine recognized changes in the polarity of the timing lead, which were designated biphasic $R$ and negative $R$. This finding correlated well with the children who had right ventricular hypertrophy. At the same time, no high degree of false positives were obtained. At the present time we believe the ECG circuitry enhances the total capabilities of the unit and lends useful information.
Some difficulties were encountered in operating the machine when there was heavy respiration and movement. These necessitated deleting the second right intercostal area from the analysis pattern. Movement and respiration or talking will cause rejection of the patient because of the type sounds produced. Indicator lights will then not show consistent findings but will flash in a random fashion. Also, respiration and movement can be noted by observing the atypical swing of the heartsound-input monitoring meter. Normal respiration had little or no effect on the unit's operation, but heavy respiration necessitated having the patient hold his breath for a 10 count. Ambient noise in the room did not affect the unit's operation. From a technical standpoint, operation of the unit is relatively simple. Total time per patient for placement of the electrode-microphone and analysis was approximately 2 minutes.

The initial clinical trial of the feasibility unit indicates good correlation between the cardiologist's and the electronic unit's auscultatory findings. The necessity for continuing analysis, for blind testing of large numbers of children with normal and subtly abnormal heart sounds, and for correlation with observations by other physicians is obvious. This work is in progress.

The capabilities of such a unit could be increased, and further circuitry design could be directed toward precise timing of the first and second heart sounds and toward consideration of their energy contents. Systole, for example, could be programed into various components to provide for recognition of all murmurs or it could be defined in terms of energy contents for early-mid-late systole to allow for recognition of specific murmurs (for example, crescendic, ejection, pansystolic). Specific circuitry for analysis of many variations of heart sounds and murmurs appears feasible by the method presented. Alterations in the circuitry to permit analysis of infant and adult heart sounds by this method are being considered.

\section{Summary}

In a new approach to analysis of heartsound patterns by electronic computer circuitry (analog-digital computer), heart sounds of 708 
children were analyzed by the unit's analyzing circuitry. Four hundred thirty-eight children had normal heart sounds and functional heart sounds according to the cardiologist's interpretations. The unit recorded 5.9 percent false positives and 1 false-negative case. Two hundred seventy children had heart disease or suspicious murmurs. In this abnormal group, 2 percent false negatives were recorded by the analyzing circuitry. For four of the six false negatives, biphasic $R$ or negative $R$ was recorded by the ECG timing lead. Overall, 0.4 percent false negatives were recorded for the total group of 708.

The initial study indicated that screening for abnormal or suspicious heart sounds by means of an analog-digital computer is possible. Good correlation was noted between the cardiologist's interpretations and computer analysis of specifically programed modalities of analysis, namely, systole, diastole, and splitting of the second sound.

\section{REFERENCES}

(1) Smith, J. M., et al.: Use of tape-recorded heart sounds in screening of children for heart disease. Circulation 20 : 887-896, November 1959.

(2) Miller, R. A., et al. : Detection of heart disease in children; results of a mass field trial with use of tape-recorded heart sounds. Circulation 25: 85-95, January 1962.

(3) Taranta, A., et al.: Automatic analysis of phonocardiograms. Ann NY Acad Sci 115 : 1062-1090, July 1964.

(4) Levine, S. A., and Harvey, W. P.: Clinical auscultation of the heart. Ed. 2. W. B. Saunders Co., Philadelphia, 1959.

(5) Butterworth, J. S., Chassin, M. R., McGrath, R., and Reppert, E. H. : Cardiac auscultation. Ed. 2. Grune \& Stratton, Inc., New York, 1960.

(6) McKusick, V. A. : Cardiovascular sound in health and disease. Williams \& Wilkins Co., Baltimore, 1958.

(7) Nadas, A.: Pediatric cardiology. Ed. 2. W. B. Saunders Co., Philadelphia, 1963.

(8) Ruch, T. C., and Fulton, J. F. : Medical physiology and biophysics. Ed. 18. W. B. Saunders Co., Philadelphia, 1961.

\section{New Secretary of Health, Education, and Welfare}

John W. Gardner, president of the Carnegie Corporation of New York and the Carnegie Foundation for the Advancement of Teaching since 1955, was sworn in as the new Secretary of Health, Education, and Welfare on August 18, 1965. The ceremony followed U.S. Senate approval of his nomination by President Lyndon B. Johnson.

Mr. Gardner succeeds Anthony J. Celebrezze, whose nomination to the U.S. Court of Appeals for the Sixth District was announced by the President on the same day as Mr. Gardner's nomination.

Born in Los Angeles in 1912, Mr. Gardner is an Honorary Fellow of Stanford University, where he received his A.B. and M.A. degrees. $\mathrm{He}$ received his $\mathrm{Ph} . \mathrm{D}$. degree from the University of California.

He taught psychology at Connecticut College for Women and Mount Holyoke College. In 1942 he joined the Foreign Broadcast Intelli- gence Service of the Federal Communications Commission where he served as chief of the Latin American section.

Joining the U.S. Marine Corps in 1943, Mr. Gardner was assigned to the Office of Strategic Services, with duty in Washington, D.C., Italy, and Austria.

He has at various times served as a consultant to the U.S. delegation to the United Nations, the Air Force, and the Department of Defense. He served on the special Task Force on Education which President John F. Kennedy formed in 1960 and headed the task force set up by President Johnson to make policy recommendations in that field. $\mathrm{He}$ is also chairman of the U.S. Advisory Commission on International Educational and Cultural Affairs.

Mr. Gardner joined the Carnegie Corporation in 1946 as executive associate and became its president in 1955. 